\title{
OCORRÊNCIA DE MEGÁSPOROS NO CARBONÍFERO SUPERIOR (SUBGRUPO ITARARÉ) NA PORÇÃO NE DA BACIA DO PARANÁ, ESTADO DE SÃO PAULO
}

\author{
PAULA GARCIA CARVALHO DO AMARAL ${ }^{1}$ \& FRESIA RICARDI-BRANCO ${ }^{2}$
}

\begin{abstract}
MEGASPORES FROM UPPER CARBONIFEROUS GLACIAL BEDS (IRATARÉ SUBGROUP) IN NE PORTION OF THE PARANÁ BASIN. SATO PAULO STATE. BRAZIL This research present the results of detailed systematic study about the megaspores found in the sedimentary rocks of Itararé Subgroup (Upper Carboniferous) located at the northeastern portion of Paraná Basin (São Paulo State), Brazil. The studied outcrop was deposited by subaquosos fans located at the lower portion of Itararé Subgroup with glacial influence during the Westaphlian times. Four species of megaspores are here described and illustrated: Sublagenicula brasiliensis, S. sinuata, Trileites tenuis and Calamospora sp. The species of the genus Sublagenicula are dominant. Are here discussed the relationship presents among other species of megaspores too, and temporal and spacial distribution of that four species for Paraná Basin and Gondwana.
\end{abstract}

Keywords: Megaspores, Carboniferous, Itararé Subgroup, Paraná Basin.

\begin{abstract}
Resumo No presente trabalho são apresentados os resultados obtidos a partir do estudo sistemático detalhado dos megásporos provenientes das rochas sedimentares do Subgrupo Itararé (Carbonífero Superior) aflorantes na porção nordeste da Bacia do Paraná, município de Campinas, Estado de São Paulo. As rochas do afloramento estudado foram depositadas por um sistema de leques subaquosos localizado na porção inferior do Subgrupo Itararé, com influencia glacial durante o westphaliano. Quatro espécies são aqui descritas e ilustradas: sublagenicula brasiliensis, s. Sinuata, trileites tenuis e calamospora sp. Destas, as duas espécies de sublagenicula são dominantes. São igualmente discutidas as relações com outras espécies de megásporos, e documentada a distribuição temporal e espacial das quatro espécies na Bacia do Paraná e no Gondwana.
\end{abstract}

Palavras-chave: Megásporos, Carbonífero, Subgrupo Itararé, bacia do Paraná.

INTRODUÇÃO O objetivo do presente trabalho foi o estudo dos megásporos obtidos em amostras de superfície coletadas em um afloramento inédito do Subgrupo Itararé, situado no Município de Campinas, SP. Também foram estudadas as relações da assembléia encontrada com outras ocorrências de megásporos no Neopaleozóico da bacia do Paraná e do Gondwana.

No total foram estudados 250 espécimes de megásporos. O afloramento apresenta uma extraordinária abundância de megásporos, ocorrendo os mesmos em grandes concentrações, nos cinco níveis estudados. Foram identificadas as espécies Sublagenicula brasiliensis, S. sinuata, Trileites tenuis e Calamospora sp., sendo as duas primeiras as mais frequientes. Além desses foram encontradas impressões de caulídeos férteis de Briófitas e caules de tamanhos variados. Assim a assembléia descrita constitui uma associação inédita para o Neocarbonífero da Bacia do Paraná.

Foi realizado em paralelo ao estudo dos megásporos, o estudo palinológico, tendo sido encontrada uma associação composta principalmente pelos gêneros Punctatispotes, Psomospora, Cristatisporites, Lundbladispora, Vallatisporites, Raistrickia, Plicatisporites, Potoniesporites e Limitisporites. A associação palinológica sugere uma idade westphaliana, corroborada por
Souza (2000) autor que incluiu o afloramento estudado dentro da Palinozona de Biointervalo Ahrensisporites cristatus.

O afloramento foi inicialmente citado e estudado faciologicamente por Souza Filho (1986), contudo os níveis aqui estudados foram re-expostos (ano 1999) em virtude de trabalhos de abertura do prolongamento da rodovia SP - 348 (Fig. 1). Tais níveis encontram-se em ambos os lados da rodovia, de forma que foram coletadas amostras de ambos os lados do afloramento.

LOCALIZAÇÃO E GEOLOGIA O afloramento localiza-se no km 96 da rodovia dos Bandeirantes (SP- 348), Município de Campinas. Como exposto anteriormente trata-se de uma exposição com aproximadamente $17,00 \mathrm{~m}$ de altura, produto de trabalhos de ampliação da rodovia, em ambos os lados da rodovia (Fig. 1).

Do ponto de vista geológico, Souza Filho (1986) realizou um mapeamento geológico (litoestratigráfico, estrutural e faciológico) do Subgrupo Itararé, na quadrícula de Campinas, Município de Campinas, SP, e reconheceu as seguintes unidades litoestratigráficas informais na área: Unidade I - arenitos em granodecrescência ascendente; Unidade II - lamitos; Unidade III - lamitos e diamictitos; Unidade IV - arenitos; Unidade V - diamictitos e arenitos; Unidade VI - arenitos com marcas onduladas e Unidade VII - diamictitos

I - Pós-graduação Geologia Sedimentar. Instituto de Geociências. Universidade de São Paulo. Rua do Lago 562. E-mail: pamaral@usp.br, Cep. 05508-080, S. Paulo, Brasil.

2 - Caixa P. 6152. D. Geologia e Recursos Minerais, Instituto de Geociências, Universidade Estadual de Campinas, Campinas, E-mail: fresia@ige.unicamp.br, CEP. 13083-970, Campinas, Brasil. 


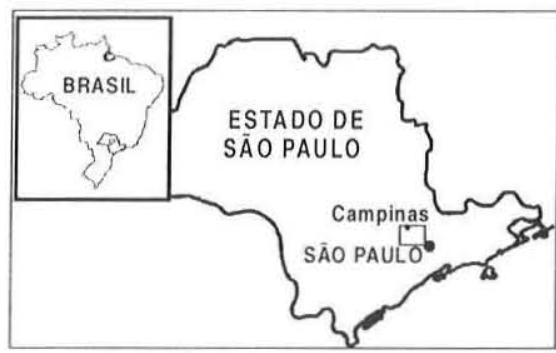

\section{LEGENDA}
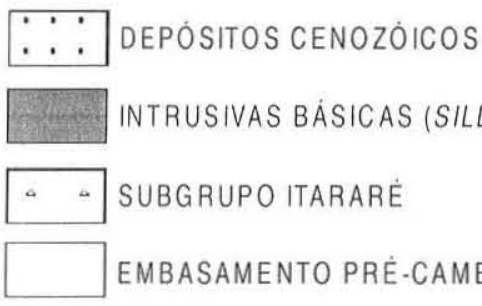

INTRUSIVAS BÁSICAS (SILLS)

SUBGRUPO ITARARE

EMBASAMENTO PRÉ-CAMBRIANO

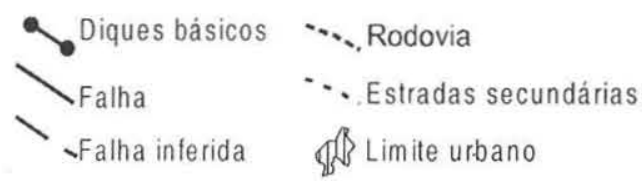

Afloramento estudado

1. Rodovia dos Bandeirantes $\mathrm{km} 96$ Campinas (SP)

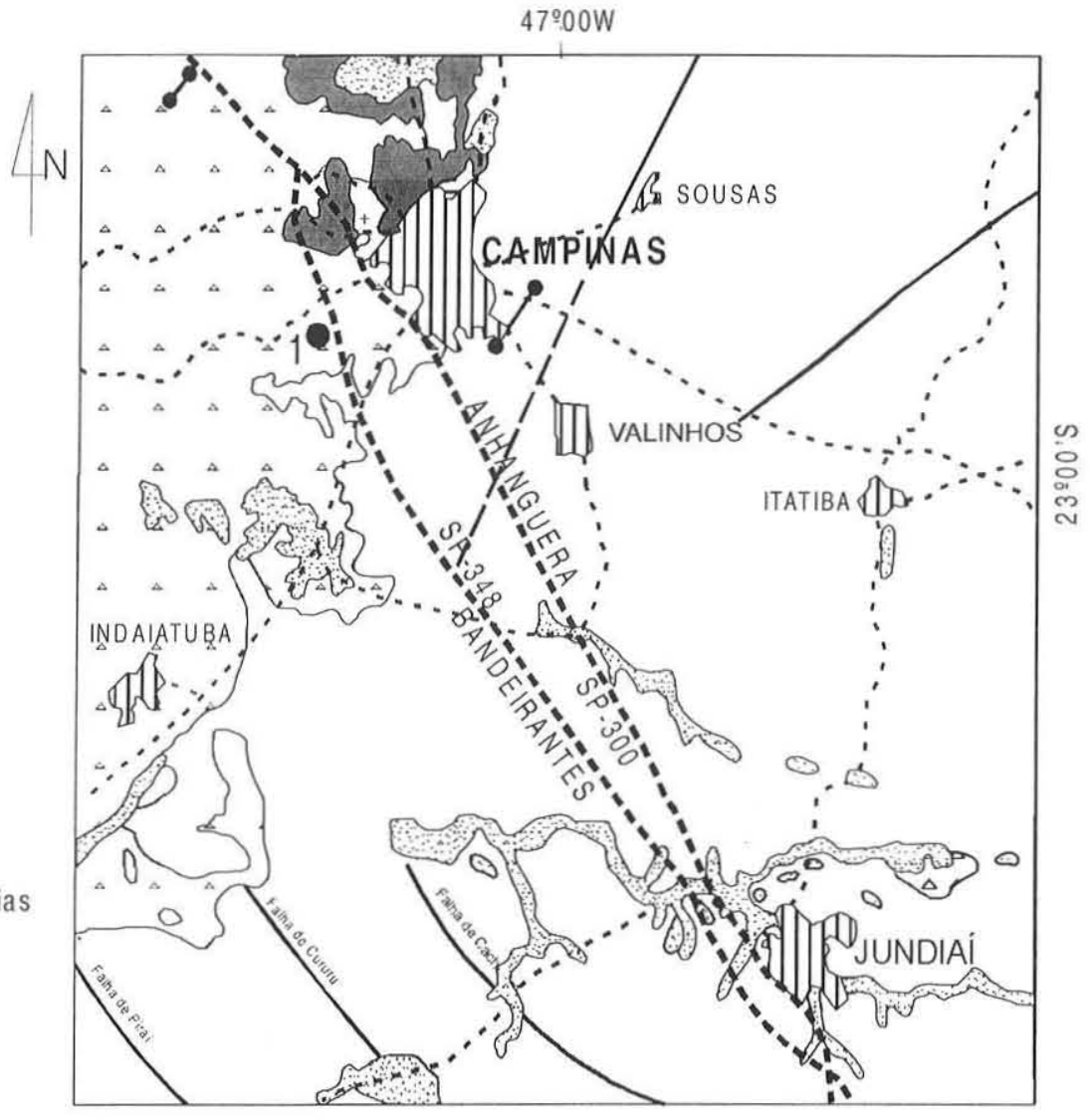

\begin{tabular}{llrr}
$0 \quad 10$ & 15 & $20 \mathrm{~km}$ \\
\hline
\end{tabular}

Figura I - Mapa geológico e de localização do Afloramento km 96 rodovia dos Bandeirantes, Campinas, Estado de São Paulo. (Modificado: Mapa Geológico do Estado de São Paulo - IPT 1981).

vermelhos.

Segundo o autor citado, o afloramento do $\mathrm{km} 96$ da rodovia dos Bandeirantes está inserido na Unidade II. Esta corresponde litologicamente a associações de lamitos maciços, acamados e ritmitos (porção inferior), siltitos e folhelhos (porção superior), sequencia posicionada na parte inferior do Subgrupo Itararé, aflorando na rodovia dos Bandeirantes $\mathrm{km} 96 \mathrm{e} 97$, bem como nas proximidades de Hortolândia, SP. Segundo Souza Filho (1986) a fauna marinha fóssil de Hortolândia, composta por Phestia, Nuculopsis, Edmondia (?) e foraminíferos indeterminados, citada por Rocha-Campos \& Rösler (1978) está contida nessa unidade.

O contato inferior da Unidade II verifica-se diretamente sobre o embasamento cristalino e interdigita-se lateralmente (em sua porção inferior de lamitos rítmicos) com a Unidade I, a qual recobre com sua porção superior de siltitos e folhelhos. O contato superior com a Unidade III é gradacional. Essas relações de contato foram descritas e podem ser observadas em Souza Filho (1986).

O mapeamento faciológico da quadrícula de Campinas elaborado por Souza Filho (1986) permitiu a individualização de um grande número de litofácies, agrupadas em fácies de arenitos, fácies de conglomerados, fácies de diamictitos, fácies de lamitos e fácies de calcários. Apesar dessas fácies ocorrerem em diversas posições estratigráficas e em diferentes unidades litoestratigráficas, o seu agrupamento em associações permitiu a interpretação de seis sistemas deposicionais, cuja evolução originou o trato deposicional hoje preservado sob o nome de Subgrupo Itararé. Foram identificados dois sistemas glaciais, o Sistema Subglacial - proglacial de Campinas e o Sistema Supraglacial de Base Úmida de Monte Mor, o Sistema de Leques Subaquosos da rodovia dos Bandeirantes, o Sistema de Plataforma de Hortolândia, o Sistema Deltáico da rodovia do Açúcar, o Sistema de Leques Aluviais de Casa Branca e o Sistema Costeiro de Tupi.

Ainda segundo Souza Filho (1986), as sequiências dos km 96 e 97 da rodovia dos Bandeirantes são interpretadas como fácies de leque subaquosos distais dentro do modelo de Walker (1978, 1980 apud Souza Filho 1986), desenvolvidos em declives altos devido a desníveis tectônicos ou, mais provavelmente, a formas de erosão glacial e alimentados por correntes de degelo.

As novas exposições deste afloramento que foram observadas no presente estudo permitiram a elaboração de uma coluna estratigráfica detalhada (Fig. 2). O perfil mostra a alternâncias, de aspecto rítmico, de camadas centimétricas a decimétricas de lamito cinza escuro maciço e arenitos bege muito finos com acamamento plano-paralelo, na base da seção. O contato entre estas camadas é brusco e não erosivo. Na parte inferior do perfil, é freqüente a presença de bioturbações que se tornam menos intensas rumo ao 


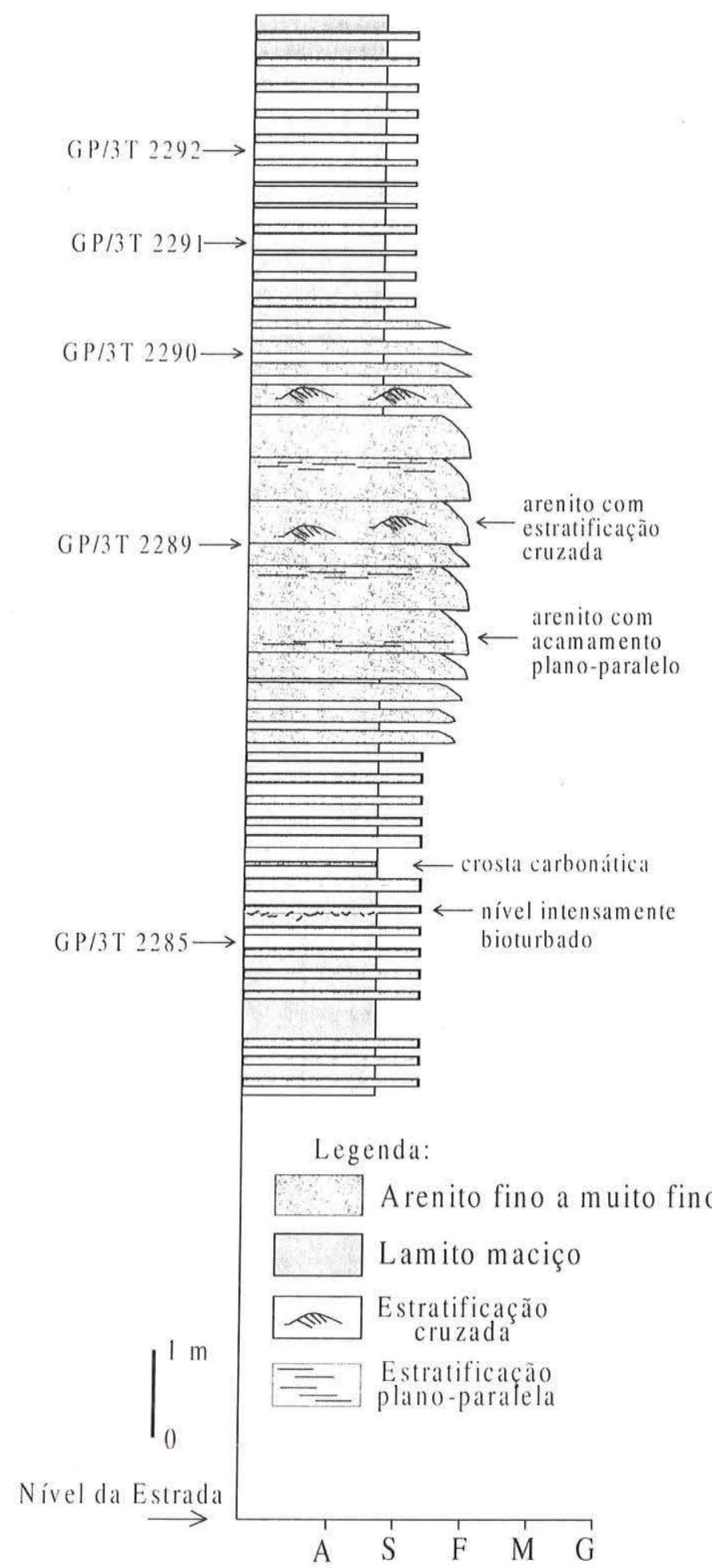

\begin{tabular}{|c|c|c|}
\hline \multirow{5}{*}{$\begin{array}{c}\text { Amostra } \\
\text { GP/3T } 2293 \\
?\end{array}$} & Espécies encontradas & $\mathrm{N}$ \\
\hline & Sublagenicula brasilicusis & () \\
\hline & Sublagenicula sinuala & 1 \\
\hline & Calamospora sp. & 1 \\
\hline & Trileites tenuis & 0 \\
\hline \multirow{5}{*}{$\begin{array}{c}\text { A mostra } \\
\text { GP/3T } 2292\end{array}$} & Espécies encontradas & $\mathrm{N}$ \\
\hline & Sublagenicula brasiliensis & 0 \\
\hline & Sublagenicula sinuata & 1 \\
\hline & Calamospora sp. & 0 \\
\hline & Trileites tenuis & 0 \\
\hline \multirow{5}{*}{$\begin{array}{c}\text { A mostra } \\
\text { GP/3T 2291 }\end{array}$} & Espécies encontradas & $\mathrm{N}$ \\
\hline & Sublagenicula brasiliensis & 27 \\
\hline & Sublagenicula sinuala & 10 \\
\hline & Calamospora sp. & 7 \\
\hline & Trileites tenuis & 2 \\
\hline \multirow{5}{*}{$\begin{array}{c}\text { A mostra } \\
\text { GP/3T } 2290\end{array}$} & Espécies encontradas & $\mathrm{N}$ \\
\hline & Sublagenicula brasiliensis & 9 \\
\hline & Sublagenicula sinuata & 6 \\
\hline & Calamospora sp. & 8 \\
\hline & Trileites tenuis & 4 \\
\hline \multirow{5}{*}{$\begin{array}{c}\text { A mostra } \\
\text { GP/3T } 2289\end{array}$} & Espécies encontradas & $\mathrm{N}$ \\
\hline & Sublagenicula brasiliensis & 6 \\
\hline & Sublagenicula sinuala & 5 \\
\hline & Calamospora sp. & 1 \\
\hline & Trileites tenuis & () \\
\hline \multirow{5}{*}{$\begin{array}{c}\text { A mos la } \\
\text { GP/3T } 2288 \\
?\end{array}$} & Espécies encontradas & $\mathrm{N}$ \\
\hline & Sublagenicula brasiliensis & 4 \\
\hline & Sublagenicula sinuala & 1 \\
\hline & Calamospora sp. & 3 \\
\hline & Trileites tenuis & 2 \\
\hline \multirow{5}{*}{$\begin{array}{c}\text { A mostra } \\
\text { GP/3T } 2287 \\
?\end{array}$} & Espécies encontradas & $\mathrm{N}$ \\
\hline & Sublagenicula brasiliensis & 4 \\
\hline & Sublagenicula sinuata & 2 \\
\hline & Calamospora sp. & 0 \\
\hline & Trileites tenuis & 1 \\
\hline \multirow{5}{*}{$\begin{array}{c}\text { A mostra } \\
\text { GP/3T } 2286 \\
?\end{array}$} & Espécies encontradas & $\mathrm{N}$ \\
\hline & Sublagenicula brasiliensis & 10 \\
\hline & Sublagenicula sinuata & 2 \\
\hline & Calamosporasp. & () \\
\hline & Trileites tenuis & 0 \\
\hline \multirow{5}{*}{$\begin{array}{c}\text { A mostra } \\
\text { GP/3T } 2285\end{array}$} & Espécies encontradas & $\mathrm{N}$ \\
\hline & Sublagenicula brasiliensis & 504 \\
\hline & Sublagenicula sinuala & 34 \\
\hline & Calamospora sp. & 36 \\
\hline & Trileites tenuis & 9 \\
\hline
\end{tabular}

Figura 2 - Seção colunar do Afloramento km 96 da rodovia dos Bandeirantes, Campinas, Estado de São Paulo e relação de espécies de megásporos encontradas em cada uma das amostras. A - argila, S - silte, Areia F - fina, $M$ - média e $G$ - grossa.

topo da seção. As camadas de arenito tornam-se mais espessas na porção mediana do perfil, apresentando acamamento planoparalelo ou estratificações cruzadas. No topo do afloramento voltam a ocorrer alternância de camadas centimétricas a decimétricas de arenitos muito finos com estratificações plano-paralelas e lamitos maciços. Ao longo do perfil, nota-se vários níveis do afloramento com abundante conteúdo de megásporos, e níveis com impressões de macrofósseis vegetais.

Ocorrências neopaleozóicas de megásporos na porção brasileira 
Tabela I - Megásporos estudados na porção brasileira da Bacia do Paraná e suas ocorrências em outras bacias do Gondwana, no intervalo Neocarbonífero - Eopermiano (modificado de Ricardi - Branco 1998). Legenda: ICS = Subgrupo Itararé, Neocarbonífero (Westfaliano). BA = Subgrupo Guatá. Formação Rio Bonito, Eopermiano (Sakmariano - Artinskiano). BH= Subgrupo Guatá. Formação Rio Bonito, Eopermiano (Artinskiano - Kunguriano). PN= Formação Palermo, Eopermiano (Kunguriano). NI= Formação El Imperial, Neocarbonífero. NC=formações Agua Colorada, Tupe, Guancodol e Sta. Máxima, Neocarbonífero. ON= Formação Ordoñez. Neopaleozóico. GP= Formação Rio Genoa, Eopermiano. Barro Branco** = megásporos encontrados por Dijstra $(1959 ; 1960)$ e Pant \& Sivrastava (1962) referidos a essa camada de carvão, com procedência duvidosa. $E P=$ Eopsermiano. $0=$ este estudo. $I=$ Trindade $(1970) .2=$ Ricardi-Branco et al. $(2002) .3=$ Arai \& Rösler (1984). 4 = Dijkstra (1955). Trindade (1959b; 1960) e Pant \& Srivastava (1962). $5=$ Trindade (1964). $6=$ Trindade (1966). 7 = Trindade (1962). 8 = Dijkstra (1955), Pant \& Srivastava (1962) e Marques-Toigo et al. (1975). $9=$ Cauduro \& Zingano (1965) e Bortoluzzi \& Veiga (1981). 10 = Garcia (1995). 11 = Cúneo et al. (1991). 12 = Spinner (1969) e Garcia (1995). $13=$ Archangelsky et al. (1989). $14=$ Pierrat \& Dijkstra (1961). $15=$ Bose \& Kar (1967). 16= Bharadwaj \& Tiwari (1970). G = Trindade (1959a; 1959b; 1960), Trindade \& Sommer (1966), Bharadwaj \& Tiwari (1970) e Dybová-Jachowicz et al. (1987).

\begin{tabular}{|c|c|c|c|c|c|c|c|c|c|c|c|c|c|c|c|c|c|c|}
\hline PAIS & \multicolumn{11}{|c|}{ BRASIL } & \multirow{2}{*}{\multicolumn{4}{|c|}{ ARGENTINA }} & \multirow{3}{*}{\begin{tabular}{|c} 
ZAIRE \\
殅
\end{tabular}} & \multirow{3}{*}{$\begin{array}{c}\text { CONGO } \\
\text { 茫 }\end{array}$} & \multirow{3}{*}{$\begin{array}{r}\text { ÍNDIA } \\
\text { 啇 }\end{array}$} \\
\hline ESTADO & \multicolumn{3}{|c|}{ SP } & \multicolumn{2}{|c|}{ PR } & \multicolumn{3}{|c|}{\begin{tabular}{|l|l}
$\mathrm{SC}$ & \\
\end{tabular}} & \multicolumn{3}{|l|}{ RS } & & & & & & & \\
\hline $\begin{array}{l}\text { Posiçào litoestratigráfica } \\
\text { e idade }\end{array}$ & $\tilde{U}$ & $\underline{U}$ & $\tilde{0}$ & §ీ & § & 䀅 & I & 丞 & I & 焉 & $z$ & $\bar{z}$ & Z & z & जे & & & \\
\hline Número de referência & 0 & 1 & G & 2 & 3 & 4 & 5 & 6 & 7 & 8 & 9 & 10 & 11 & 12 & 13 & 14 & 15 & 16 \\
\hline 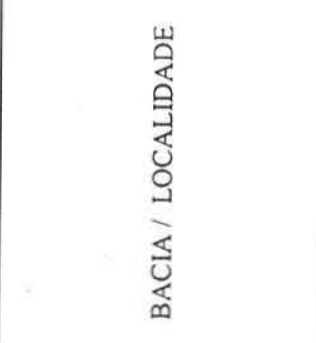 & 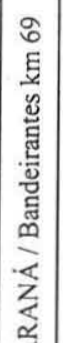 & 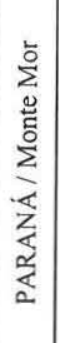 & 焉 & 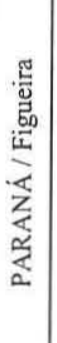 & 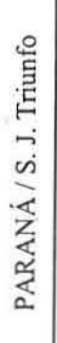 & 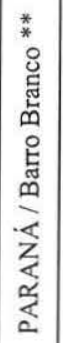 & 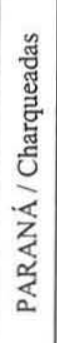 & 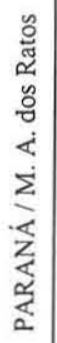 & 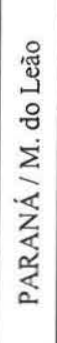 & 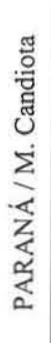 & 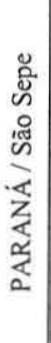 & 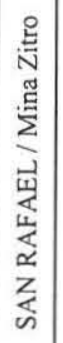 & 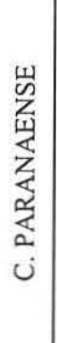 & 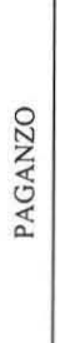 & 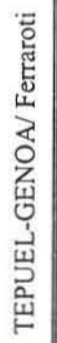 & 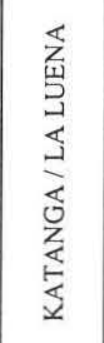 & 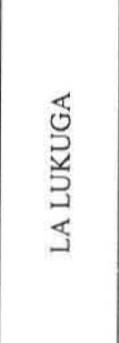 & 采 \\
\hline MEGÁSPOROS & & & & & & & & & & & & & & & & & & \\
\hline Sublagenicula brasiliensis & & & & & & & & & tit & 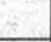 & & & +5 & & & & t. & \\
\hline S. brasiliensis var. minor & & & & & & & & & & & & & & & & & & \\
\hline S. cf. S. brasiliensis & & & & & & & & & & & & & & & & & & \\
\hline Sublagenicula sinuata & & & & & & & & & & & & & & & & & & \\
\hline Lagenoisporites triunfensis & & & & & & & & & & & & & & & & & & \\
\hline L. scutiformis & & & & & & & & & & & & & & & & & & \\
\hline Lagenoisporites tripartites & & & & & & & & & & & & & & & & & & \\
\hline L. candiotensis & & & & & & & & & & tat & & & & & & & & \\
\hline Lagenoisporites sp. A & & & & & & & & & & & & & & & & & & \\
\hline Lagenoisporites spp. & & & & & & & & & & & & & & & & & & \\
\hline Trileites labiosus & & $\mathrm{de}$ & & & & -1 & \pm 6 & & & & & & & & & & & \\
\hline Trileites nitens & & & & & & 1 & 40 & & & tas & 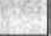 & & & & & & & \\
\hline Trileites? nitens & & & & & & & & & & & & & & & & & & \\
\hline Trileites trivalis & & & & & & & & & & & & & & & & & & \\
\hline Trileites temuis & & & & & & 20 & 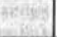 & & & 4 & & & & (206) & & & & \\
\hline Trileites vulgatus & & & & & & & & & & & & & & & & & & \\
\hline Duosporites trivedii & & & & & & & & & & & & & & & & & & \\
\hline Trileites endosporitiferus & & & & & & & & & & & & & & & & & & \\
\hline Setosisporites furcatus & & & & & & & & & & & & & & & & & & \\
\hline S. cf. S. furcatus & & & & & & & & & & & & & & & & & & \\
\hline Setosisporites sp. & & & & & & & & & & & & & & & & & & \\
\hline Cystosporites sp. & & & & & & & & & & & & & & & & & & \\
\hline Calamospora sp. & 1 & a & & & & & a & & & a & & & & & & & & \\
\hline
\end{tabular}


da Bacia do Paraná Na porção oriental da Bacia do Paraná, cuja faixa aflorante estende-se pelos estados de São Paulo, Paraná, Santa Catarina e Rio Grande do Sul, os megásporos ocorrem freqüentemente associados a estratos e/ou camadas de carvão, do Paleozóico Superior, gerados, principalmente, em ambientes deltaicos durante episódios interglaciais e pós-glaciais. Os registros mais antigos encontrados, até o desenvolvimento desta pesquisa, para a porção oriental (Estado de São Paulo) da bacia, eram Monte Mor e Buri (Tabela I), no Subgrupo Itararé (Neocarbonífero).

Em Monte Mor, os megásporos ocorrem junto a uma variada tafoflora de macrofósseis vegetais (Millan 1972, 1987, Mune \& Bernardes-de-Oliveira 2002) composta principalmente por licófitas, (Brasilodendron, Bumbudendron, entre outros), esfenófitas (Sphenophyllum sp., Paracalamites montemorensis, P. Levis, P. australis, etc.), Pteridófilas/progimnospermópsidas (Botrychiopsis sp. A, Botrychiopsis sp. B, Eusphenopteris sp., Nothorhacopteris cf. N. sp. A, Nothorhacopteris cf. N. sp. B), Gimnospermas (cf. Ginkgophyllum sp., Noeggerathiopsis hislopii, Buriadia sp., Paranocladus ?fallax, Samarosis seixasii e Cordaicarpus barbosanus), estando macro e microfósseis associados a uma camada de carvão.

As espécies de megásporos encontradas por Trindade (1970), associadas a camada de carvão de Monte Mor foram: Lagenoisporites brasiliensis, L. sinuatus, L. scutiformis, L. tripartites, Setosisporites sp., Trileites labiosus, T. endosporitifeus, Calamospora sp. e Duosporites trivalis, que documentam a presença de vários gêneros de licópsidas (Lagenoisporites, Setosisporites, etc), e um de esfenópsida (Calamospora). As espécies dominantes em Monte Mor são Sublagenicula brasiliensis e Lagenoisporites scutiformis. Para Buri são indicadas por Trindade (1960) a presença de Sublagenicula brasiliensis, $S$. sinuatus e Trileites nitens.

Nos estados do Paraná e Santa Catarina, os megásporos encontram-se associados a camadas de carvão da Formação Rio Bonito (Subgrupo Guatá) já no Eopermiano. As ocorrências mais antigas foram descritas para o Estado do Paraná em camadas pertencentes ao Membro Triunfo (?Sakmariano-Artinskiano), sendo encontradas nas localidades de São João do Triunfo (Arai \& Rösler 1984) e Figueira (Ricardi-Branco 1998 e Ricardi-Branco et al. 2002). Nessas assembléias as espécies dominantes são Lagenoisporites triunfensis e Lagenoisporites scutiformis. Contudo, essas assembléias são menos ricas em espécies do que aquela de Monte Mor. Esse fato pode estar associado a uma menor diversificação específica de licópsidas presentes (Tabela 1).

Em Santa Catarina, megásporos foram encontrados no membro superior da Formação Rio Bonito (Membro Siderópolis, Artinskiano-Kunguriano), tendo sido estudada somente a assembléia de Barro Branco (Dijkstra 1955, Trindade 1959be 1960, Pant \& Srivastava 1962). Essa assembléia apresenta-se mais rica em espécies do que as do Paraná, possivelmente relacionada com o melhoramento das condições climáticas no decorrer do Permiano (Tabela 1).

No Rio Grande do Sul, foram estudadas quatro assembléias de megásporos com idade artinskiana/kunguriana, na Formação Rio Bonito, sendo Charqueadas (Trindade 1964) e Candiota (Dijkstra 1955, Pant \& Srivastava 1962, Marques-Toigo et al. 1975), as mais diversificadas em espécies. Ainda nesse estado, na localidade de São Sepé, Bortoluzzi \& Veiga (1981) e Cauduro \& Zingano (1965) estudaram megásporos em camadas da Formação Palermo (Kunguriano), topo do Grupo Tubarão (Tabela 1).
MATERIAL E MÉTODOS As amostras estudadas provem de cinco níveis diferentes do afloramento, conforme observado no perfil estratigráfico da figura 2. As amostras 2286, 2287, 2288 e 2293 foram encontradas no rejeito próximas aos patamares dos níveis estudados. Os megásporos foram retirados da rocha mediante a técnica de maceração em blocos. Essa técnica consiste em dissolver pequenos blocos de rocha contendo megásporos na sua superfície, por submersão em ácido fluorídrico (HF aproximadamente 20\%), até os megásporos se desprenderem da matriz. Logo o resíduo é lavado como água destilada varias vezes, sendo posteriormente coletados com a ajuda de um pincel e montados em lâminas secas.

Foram observados cerca de 700 exemplares de megásporos incarbonizados, dos quais 250 foram montados em lâminas secas, estudados sob estereomicroscópio, descritos, desenhados e identificados por comparação bibliográfica. Os espécimes mais representativos de cada uma das espécies encontradas foram fotografados.

O material, está catalogado na Coleção Tipo (3T) do Laboratório de Sistemática Paleontológica do Departamento de Geologia Sedimentare Ambiental, Instituto de Geociências /USP, e compreende amostras : GP/3T 2285 a 2293.

Sistemática Paleontológica Foi seguida a classificação para megásporos gulados sugerida por Dybová-Jachowicz et al. (1979 e 1987), tendo sido mantida a sistemática tradicional de Potonié (1956) e Piérart (1975).

Anteturma Megasporites Pant, 1962

Turma Triletes (Reinsch) Potonié et Kremp,1954

Suprasubtuma Lagenotriletes Potonié et Kremp, 1957

Subturma Gulati Bharadwaj, 1957

Gênero Sublagenicula (Potonié et Kremp) Dybová-Jachowicz, Jachowicz, Karczewska, Lachkar, Loboziak, Piérart, Turnau et Zoldani, 1979

Sublagenicula brasiliensis (Dijkstra) Dybová-Jachowicz, Jachowicz, Karczewska, Lachkar, Loboziak, Piérart, Turnau et Zoldani, 1979. Figura 3A-D. Amostras estudadas: GP/3T 2285, 2286, 2287, 2288, 2290e 2291. Lâminas: 1-7e 9

DESCRIÇÃO Megásporos triletes, subgulados, prolados em compressão lateral e ovais em compressão próximo-distal. Raios triletes retos. Cristas arcuadas, geralmente, bem definidas. Confluência dos raios triletes com as cristas arcuadas, por vezes, definidas por pequenas aurículas triangulares. Ornamentação da área de contato psilada, e da área distal psilada a escabrada.

Dimensões:vista proximal distal, total de exemplares medidos 37. Comprimento $444-1400 \mu \mathrm{m}$ e largura $422-1333 \mu \mathrm{m}$. Raio trilete 289-689 $\mu \mathrm{m}$. Crista arcuata 355-933 $\mu \mathrm{m}$. Vista lateral: total de exemplares medidos 74. Comprimento 533-1555 $\mu$ m e largura 466- 1400 $\mu \mathrm{m}$. Raio trilete $266-778 \mu \mathrm{m}$. Crista arcuata $289-1111 \mu \mathrm{m}$.

DISCUSSÃO E COMENTÁRIOS Nem todas as características puderam ser observadas em todos os espécimes. As cristas arcuadas, por exemplo, em alguns exemplares não estavam bem definidas, impossibilitando a determinação do seu tamanho. Contudo foram encontrados, em sete das amostras, um total de 564 espécimes com as características morfológicas acima descritas, dos quais 114 desses foram estudados detalhadamente (descritos, desenhados e medidos), os demais apenas separados e contados. Acredita-se que esse número de espécimes descritos seja 
A

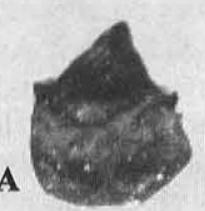

C

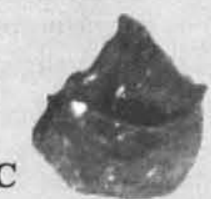

I
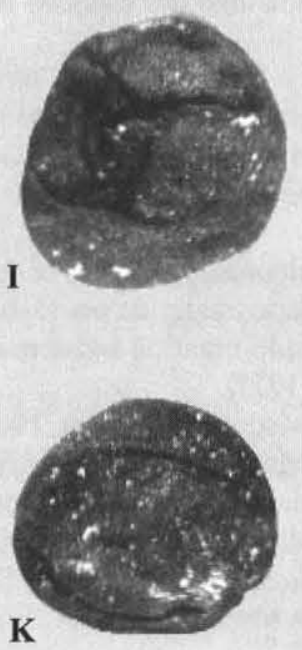
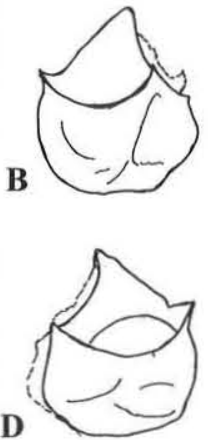

D

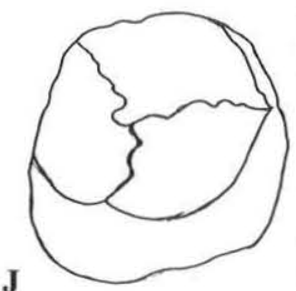

J

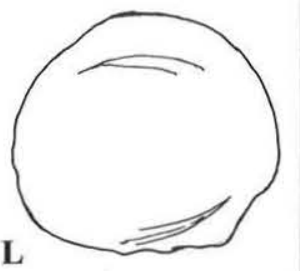

$\mathbf{E}$

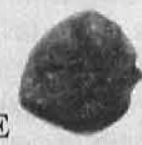

F

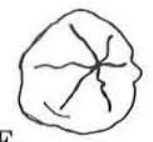

G

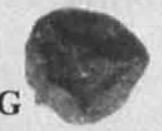

H

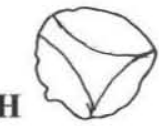

N

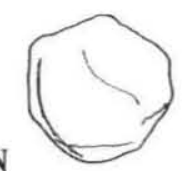

o

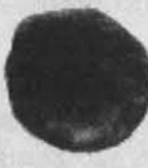

M
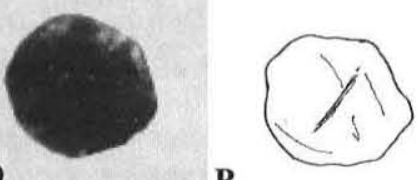

Figura 3 - Megásporos $($ Escala $=1 \mathrm{~mm}$ ). A - Sublagenicula brasiliensis compressão lateral (vista anterior, GP/3T 2291). B Desenho esquemático de A. C - Sublagenicula brasiliensis compressão lateral (vista posterior, 2285).. D - Desenho esquemático de CE-Trileites tenuis compressão próximo-distal (vista anterior, 2290). Notar o padrão actinomorfo das pregas da exina. F - Desenho esquemático de E. $G$ - Trileites tenuis compressão distal (vista posterior, 2290). H - Desenho esquemático de G. I - Sublagenicula sinuata compressão próximo-distal (vista anterior, 2285). Notar raios triletes sinuosos. J-Desenho esquemático de I. K - Sublagenicula sinuata compressão distal (vista posterior, 2285). L - Desenho esquemático de K. M - Calamospora sp. compressão lateral (vista anterior; 2285). $N$ - Desenho esquemático de M. O - Calamospora sp. - compressão lateral (vista posterior, 2285). $P$ - Desenho esquemático de $O$.

suficientemente grande e que englobe todas as variações intraespecíficas de megásporos dessa espécie. Na amostra GP/3T 2289 foi observado um exemplar com a gula aberta, o que poderia indicar que esse germinou.

$\mathrm{Na}$ maioria dos megásporos estudados pertencentes a esta espécie a exina do hemisfério distal apresentava ornamentação psilada; apenas em quatorze espécimes a exina mostrava-se levemente escabrada.

Os espécimes descritos assemelham-se com a espécie de megásporos Sublagenicula brasiliensis (Dijkstra) DybováJachowicz et al., no que se refere à gula, raio trilete, ornamentação da exina da área distal, área de contato e dimensões. Pode-se observar que essa espécie apresenta grande variação intraespecífica, assim como indicado em Trindade (1959b, 1962).

Foram encontrados três exemplares de dimensões menores que o limite inferior da espécie (amostras GP/3T 2290 e 2291), esses não entraram nos cálculos da frequêencia de dimensões acima apresentadas. Estes espécimes podem corresponder a formas abortadas, conforme indicado por Dijkstra (1955).

No Estado de São Paulo, Sublagenicula brasiliensis ocorre nas assembléias de megásporos de Monte Mor e Buri, do Subgrupo Itararé, ambas neocarboníferas e estudadas por Trindade (1959b e 1970). Assim como indicado por aquela autora para Monte Mor e Buri, Sublagenicula brasiliensis é a forma dominante dentro das assembléias de megásporos estudadas, constituindo no caso de Monte Mor $80 \%$ dos exemplares descritos.

Fora do Estado de São Paulo, mas ainda na porção brasileira da Bacia do Paraná, esta espécie foi encontrada nos estados do Paraná(Ricardi-Branco et al. 2002), Santa Catarina (Dijkstra 1955, Trindade, 1959b e 1960, Pant \& Srivastava 1962) e Rio Grande do Sul (Dijkstra 1955, Trindade 1962, 1964 e 1966, Pant \& Srivastava 1962, Marques-Toigo et al. 1975, Cauduro \& Zingano 1965, Bortoluzzi \& Veiga 1981).

Fora do Brasil (Tabela 1), esta espécie ocorre na América do 
Sul, no Carbonífero Superior da Argentina. Para o continente africano esta tem sido encontrada no Carbonífero Inferior do Egito e Tchad; no Viseano da Nigéria; no Namuriano da Argélia e oeste da Líbia e no Permiano Inferior de Zaire e da República Democrática do Congo (Dybová-Jachowicz et al. 1987).

Sublagenicula sinuata (Dijkstra) Dybová-Jachowicz Jachowicz, Karczewska, Lachkar, Loboziak, Piérart, Turnau et Zoldani 1979

Figura 3, I - L. Amostras estudadas:GP/3T 2285, 2286, 2287, 2288, 2289, 2290, 2291, 2292 e 2293. Lâminas: 1, 3-6, 8-9

DESCRIÇÃO Megásporos triletes, subgulados, na sua maioria, em compressão próximo-distal, forma circular a oval. Cristas arcuadas freqüentemente bem marcadas. Raios triletes ondulados e sinuosos. Ornamentação da área de contado psilada, e do hemisfério distal psilada a escabrada.

Dimensões: vista proximal distal: total de exemplares medidos 60. Comprimento 466-1444 $\mu \mathrm{m}$ e largura 444-1378 $\mu \mathrm{m}$. Raio trilete $266 \mu \mathrm{m}$. Crista arcuata $444-711 \mu \mathrm{m}$. Vista lateral, total de exempalres medidos 2. Comprimento 778-1022 $\mu$ me largura 866-955 $\mu \mathrm{m}$. Raio trilete 511-578 $\mu \mathrm{m}$. Crista arcuata 600-666 $\mu \mathrm{m}$.

DISCUSSÃO E COMENTÁRIOS Foram estudados 62 espécimens de Sublagenicula sinuata, representando 9\% da assembléia estudada. A maioria dos exemplares descritos encontram-se em compressão próximo-distal e possuem a ornamentação da exina psilada. Apenas dois espécimes (amostras GP/3T 2290 e 2291) foram encontrados em compressão lateral e seis com ornamentação da exina do hemisfério distal escabrada. Foi observado também, na amostra GP/3T 2292, um exemplar com a gula aberta, podendo ser um indicativo que esse teria germinado.

Os espécimes encontrados assemelham-se a Sublagenicula sinuata no que se refere ao tipo de gula, raios triletes, área de contato e tipo mais freqüente de compressão. Dijkstra (1955) e Dybová-Jachowicz et al. (1987) mencionam a presença de grânulos ou báculas na exina do hemisfério distal, características não observadas, em espécime algum estudado nesse trabalho. Entretanto, Dybová-Jachowicz et al. (1979) mencionam que muitas vezes essas báculas podem ser perdidas durante o transporte e diagênese. Nos exemplares dessa espécie descritos por Trindade (1970), para Monte Mor, essas características não foram observadas, sendo apresentados somente espécimes com exina psilada, assemelhando-se à maioria dos espécimes deste estudo

No Estado de São Paulo, a espécie Sublagenicula sinuata foi estudada por Trindade (1959b, 1970) em Monte Mor e Buri (Subgrupo Itararé, Carbonífero Superior). Em Santa Catarina, essa espécie foi descrita por Dijkstra (1955), Trindade (1959b e 1960) e Pant \& Srivastava (1962), na camada Barro Branco, Membro Siderópolis, da Formação Rio Bonito, do Eopermiano (Tabela 1).

No Estado do Rio Grande do Sul, também em camadas da Formação Rio Bonito, a referida espécie foi encontrada em: Charqueadas (Trindade 1964), na Mina Arroio dos Ratos (Trindade 1966), na Mina do Leão (Trindade 1962) e na Mina de Candiota (Dijkstra 1955, Pant \& Srivastava 1962, Marques-Toigo et al. 1975). Na Formação Palermo, foi encontrada em São Sepé (Cauduro \& Zingano 1965, Bortoluzzi \& Veiga 1981).

Fora do Brasil, ocorrências de $S$. sinuata foram registradas no Andar Barakar da Índia (Dijkstra 1955). Em comparação com Sublagenicula brasiliensis, a distribuição geográfica dessa espécie é bem mais restrita.
Suprasubtuma Azonotriletes Luber, 1935

Subturma Laevigati (Bennie et Kidston) Potonié et Kremp, 1954

Gênero Trileites (Erdtman) Potonié, 1956

Trileites tenuis (Dijkstra) Trindade, 1959

Figura 3, E-H. Amostras estudadas: GP/3T 2285, 2287, 2288, 2290 e 2291. Lâminas: 1, 3, 5-6, 8-9

DESCRIÇÃO Megásporos circulares esféricos, em compressão proximal-distal e ovais em compressão lateral. Área de contato, quando presente, de coloração mais escura que o restante do corpo do megásporo. Cristas arcuadas e raios triletes não distinguíveis, mascarados por dobras da exina. Exina fina, translúcida e psilada.

Dimensões: vista proximal: total de exemplares medidos 18. Comprimento $466-822 \mu \mathrm{m}$ e largura $400-788 \mu \mathrm{m}$.

DISCUSSÃO E COMENTÁRIOS Dos megásporos acima descritos, foram encontrados 18 exemplares, correspondendo a $3 \%$ da assembléia geral no afloramento do $\mathrm{km} 96$ da rodovia dos Bandeirantes. Cabe ressaltar que todos os espécimes estudados apresentam pregas da exina, que se iniciam na porção central do megásporo e prolongam-se até as margens. Essas pregas, quando observadas em compressão proximal-distal, sugerem um padrão actinomorfo na sua distribuição. Em dois exemplares (amostra GP/ 3T 2290) foi observada nitidamente a área de contato. Essa apresenta coloração mais escura, se comparada com o resto do corpo do megásporo.

Os megásporos descritos assemelham-se à espécie de Trileites tenuis Dijkstra, no que se refere à exina fina e translúcida, área de contato, na maioria das vezes, indistinta e no padrão actinomórfo de suas dobras. Embora a maioria dos espécimes aqui descritos possua dimensões inferiores às dos espécimes de Dijkstra (1955 / $720-1010 \mu \mathrm{m}$ ), esse parâmetro apresenta-se variável para outros autores. Pant \& Srivastava (1962) descrevem megásporos com dimensões no intervalo de $432-1960 \mu \mathrm{m}$, assumindo uma variação de tamanhos muito maior do que a encontrada por Dijkstra (1955), o que engloba as dimensões aqui encontradas.

Outro aspecto que merece ser mencionado, refere-se a problemática existente na inclusão dessa espécie no gênero Trileites Erdtman, ou dentro do gênero Duosporites Høeg, Bose \& Manum, uma vez que o segundo foi originalmente descrito com base em características do mesospório (Piérart 1959, Spinner 1969), somente observadas no estudo dos espécimes em microscópio de luz transmitida. No presente trabalho não foi utilizada essa técnica, não sendo, portanto, possível observar o mesospório. Assim, optou-se por designar os espécimes como Trileites tenuis, uma vez que essa espécie foi originalmente descrita com base em exemplares também observados sob luz refletida.

Os espécimes de $T$. tenuis aqui identificados representam a ocorrência mais antiga dessa espécie registrada, até o momento, na Bacia do Paraná, considerando-se que essa foi somente encontrada por Dijkstra (1955), Trindade (1959a e b, 1960, 1964), Pant \& Srivastava (1962) e Marques-Toigo et al. (1975) em camadas eopermianas da Formação Rio Bonito, nos Estados de Santa Catarina e Rio Grande do Sul (Tabela 1).

Fora do Brasil, T. tenuis foi encontrado estudado na Argentina (Spinner 1969) na Formação Tupe (Neocarbonífero), em Katanga, na República Democrática do Congo (Piérart \& Dijkstra 1961) e na Índia (Pant \& Srivastava, 1962).

Gênero Calamospora Schopf, Wilson \& Bentall, 1944

Calamospora sp. 
Figura 3, M-P. Amostras estudadas: GP/3T 2285, 2287, 2289, 2290, 2291 e 2293. Lâminas: 1, 3-5, 8-9

DESCRIÇÃO Megásporos triletes, ovais tanto em vista polar como em equatorial, Cristas arcuadas não distinguíveis. Raios triletes raramente observados, quando presentes, com 3/4 do raio do megásporo. Exina psilada, fina, translúcida e fortemente plicada.

Dimensões: vista proximal: número de exemplares medidos 55. Comprimento 555-911 $\mu \mathrm{m}$ e largura $422-800 \mu \mathrm{m}$. Raio trilete 266$533 \mu \mathrm{m}$.

DISCUSSÃO E COMENTÁRIOS Foram encontrados 56 espécimens da espécie acima descrita, em seis das amostras. $\mathrm{O}$ raio trilete é raramente distinguível e só foi possível observa-lo em sete dos exemplares devido ao fato dos exemplares terem a exina fortemente plicada, em decorrência dos processos diagenéticos.

Os espécimens estudados assemelham-se ao gênero Calamospora no que se refere à exina psilada, fina e translúcida, com fortes dobras secundárias e forma oval. Assim como Dijkstra (1955) e Trindade (1959a, 1970), na assembléia estudada também não foi possível a determinação específica para esses espécimes, uma vez que as espécies de Calamospora são muito semelhantes entre si. Esses autores também não puderam observar as características dos raios triletes e cristas arcuadas em seu material.

No Estado de São Paulo, o gênero Calamospora foi estudado por Trindade (1970) para Monte Mor (Carbonífero Superior do Subgrupo Itararé). Esse gênero também foi reportado para o Estado do Rio Grande do Sul por Dijkstra (1955), na Mina de Candiota, e por Trindade (1959a), em Charqueadas, ambas localidades pertencentes à Formação Rio Bonito e de idade eopermiana (Tabela 1). Fora do Brasil, Calamospora ocorre em Katanga (Zaire) e no Congo e foi estudada por Piérart \& Dijkstra (1961), esse megásporo é possivelmente aparentado com Esfenópsidas.

DISCUSSÃO DOS RESULTADOS Observando hoje uma área de exposição do afloramento do $\mathrm{km} 96$ da rodovia Bandeirantes maior do que aquela observada por Souza Filho (1986), é possível notar as mesmas litologias e estruturas, porém, é interessante ressaltar que os vários níveis do afloramento contendo megásporos em abundância não foram registrados por aquele autor, constituindo-se, no momento, em uma nova contribuição.

Foram estudadas e identificadas quatro espécies de megásporos: Sublagenicula brasiliensis, S. sinuata, Trileites tenuis e Calamospora sp., cujas distribuições quantitativas ao longo do perfil estratigráfico podem ser observadas na figura 2. Com relação à assembléia analisada em os todos os níveis estudados do afloramento essa é homogênea, sendo sempre a espécie Sublagenicula brasiliensis dominante e possível de encontrar até 504 exemplares em uma amostra, seguida por $S$. sinuata e Calamospora sp.

A ocorrência do gênero Calamospora pode sugerir a presença de esfenópsidas na paleoflora do afloramento do $\mathrm{km} 96$ da rodovia Bandeirantes. Assim como Sublagenicula brasiliensis, S. sinuata e Trileites tenuis sugerem a presença de licópsidas.

Foi registrada pela primeira vez a espécie Trileites tenuis no Estado de São Paulo, constituindo a ocorrência mais setentrional dentro da porção brasileira da Bacia do Paraná. Até o momento, esta é também a primeira vez que se observa essa espécie de megásporos não associada a camadas de carvão.

As espécies Sublagenicula brasiliensis e Sublagenicula sinucita, já tinham sido anteriormente encontradas no Subgrupo Itararé, em Monte Mor e Buri, associadas a camadas de carvão.
Como na palinoflora de Monte Mor, a espécie dominante na assembléia estudada é Sublagenicula brasiliensis, que ocorre aqui constituindo $80 \%$ dos espécimes.

A grande abundância de megásporos ocorrendo em vários níveis do afloramento é, segundo Tyson (1995), forte indicação de grande proximidade a costa, ou a redeposição de áreas fontes flúvio-deltaicas, além de transporte de pequena duração.

Em estudo palinológico realizado neste mesmo afloramento (Amaral 2000), esses sedimentos estão posicionados dentro da Palinozona de Biointervalo Ahrensisporites cristatus (?Westphaliano) Souza (2000), sendo conferida uma idade westfaliana a esses níveis. Esse posicionamento bioestratigráfico corrobora com os dados litoestratigráficos de Souza Filho (1986) quanto à sua situação na porção inferior do Subgrupo Itararé. A partir desse último dado, a assembléia de megásporos encontrada no afloramento $\mathrm{km} 96$ da rodovia dos Bandeirantes está posicionada estratigraficamente junto com as tafofloras de Monte Mor e Buri, os registros mais antigos encontrados no Estado de São Paulo, no Subgrupo Itararé (Tabela 1).

Fora do Brasil são encontradas assembléias de idade neocarbonífera com algumas espécies em comum na Argentina, sendo as assembléias brasileiras sensivelmente mais diversas em taxa que as argentinas. A espécie $S$. brasiliensis também pode ser encontrada em assembléias de megásporos do Eocarbonífero no norte da África (Chad, Líbia, Egito, etc) como indicado por autores como Piérart (1981), Dybová-Jachowcz et al. (1987) dentre outros. Para explicar a ampla distribuição temporal e espacial dessa espécie, foram levantadas diferentes hipóteses. Piérart (1981) sugeriu que a presença de um grupo de licófitas (representadas por seus megásporos) transgressivas, integrado também por Trileites tenuis e Setosisporites furcatus. Esse grupo de licófitas transgressivas, teria vindo a formar parte da Flora de Glossopteris no início do Permiano, após o fim da glaciação proveniente de áreas periféricas à glaciação do Gondwana. Cúneo et al. (1991) sugerem para $S$. brasiliensis se tratar de taxa diferentes que apresentariam padrões semelhantes de dispersão e reprodução.

Por outro lado é possível sugerir, com base nos megásporos registrados no Subgrupo Itararé (SP), que a morfo-espécie $S$. brasileinsis seja um tipo morfológico de megásporos não somente de uma espécie, senão comum a toda uma família neopaleozóica de Licopsidas, muito bem sucedida, que facilmente colonizou os ambientes hidrófilos do Neopaleozóico gondwânico.

Considerando a associação de megásporos estudada conjuntamente com o ambiente e paleoclima sugeridos para o afloramento do $\mathrm{km} 96$ da rodovia Bandeirantes, é possível sugerir a hipótese de esses taxa terem se instalado na Bacia do Paraná antes do fim da Glaciação no Westafaliano, habitando as margens da Bacia do Paraná, ainda sob a influência de um clima glacial. Essas "espécies pioneiras" (Tabela 1) continuam presentes durante períodos de melhorias climáticas relacionadas a estágios interglaciais, como no caso de Monte Mor e Buri. Durante o Eopermiano espécies de licófitas e esfenófitas (aqui inferidas por seus megásporos como S. brasiliensis - Tabela 1), se distribuíram por toda a paleolinha de costa da porção oriental da Bacia do Paraná associadas com os ambientes deltaicos geradores de camadas de carvão da Formação Rio Bonito (Ricardi-Branco et al. 2002)

Agradecimentos À FAPESP que, através do Projeto Temático 97/03639-8 - "Levantamento da composição e sucessão paleoflorística do Neocarbonífero - Eopermiano (Grupo Tubarão) do Estado de São Paulo", financiou esta pesquisa. Agradecem 
também aos Prof. Dra. Mary Bernardes de Oliveira, pela sua ajuda na pesquisa e estímulo, Prof. Dr. Paulo Roberto dos Santos pela ajuda prestada nos trabalhos de campo e elaboração da coluna estratigráfica do afloramento, ao Geol. Fábio Branco pela ajuda nos trabalhos de campo e aos relatores da RBG pelas sugestões ao manuscrito.

\section{Referências}

Amaral P.G.C. do 2000. Estudo da tafoflora do Subgrupo Itararé no afforamento km 96 da rodovia dos Bandeirantes. Municipio de Cumpinas. SP. Monografia de Trabalho de Formatura, Instituto de Geociências, Universidade de São Paulo, 39pp.

Arai M. \& Rösler O. 1984. Megásporos de São João do Triunfo, Formação Rio Bonito (Permiano). Boletim IG - USP, 15:53-64.

Archangelsky S., Cúneo R., Seoane L.V. 1989. Estudios sobre megásporas pérmicas argentinas. I. Sublagenicula brasiliensis (Dijkstra) DybováJachowicz et al. Ameghiniana, 26:209-217.

Bharadwaj D.J. \& Tiwari R.S. 1970. Lower Gondwana megaspores - A Monograph. Palaeontographica Abt. B, 129:1-70.

Bose M.N. \& Kar R.K.1967. Paleozoic sporae disperse from Congo. V. - Megaspores from Assise des Schistes noirs de La Lucuga. Ann. Musee Roy. Afr: Centrale., Sc. Géol., 54:108-111.

Bortoluzzi C.A. \& Veiga P. 1981. Considerações sobre a geologia da área dos afloramentos fossilíferos de São Sepé, RS. Pesquisas, 14:2326.

Cauduro A.D. \& Zingano A.G. 1965. Megásporos en argilete silicifié de São Sepé, Rio Grande do Sul. Anciis da Academia Brasileira de Ciências, 37: 273-282.

Cúneo R., Seoane L.V. de. Archangelsky S. 1991. Estudios sobre megásporas pérmicas argentinas. II. Sublagenicula nuda y $S$. brasiliensis de la Cuenca Chacoparanaense, Argentina. Ameghiniana, 28:55-62.

Dijkstra S.J. 1955. Some Brazilian megaspores, Lower Permian in age, and their comparision with Lower Gondwana spores from Índia. Mededelingen Geologische Strichting Nieuwe Serie, 9:5-10.

Dybová-Jachowicz S., Jachowicz A., Karczewska J., Lachkar G., Loboziak S., Piérart P., Turnau E., Zoldani E. 1979. Note préliminaire sur la revision des megaspores à guls du Carbonifère. Les principes de la classification. Acta Palaeontologica Polonica, 24:411-422.

Dybová-Jachowicz S., Jachowicz A., Karczewska J., Lachkar G., Loboziak S., Piérart, P., Turnau E., Zoldani E. 1987. Revision of carboniferous megaspores with gula (Parth three). Prace Instytutu Geologicznego, Varsóvia, 49pp.

Garcia, G. 1995. Palinológia de la Formación El Imperial, Paleozoico Superior, Cuenca de San Rafael, Argentina. Parte 1: Esporas. Ameghiniana, 32:315-33.9

IPT - Instituto de Pesquisa Tecnológicas do Estado de São Paulo; Divisão de Minas e Geologia Aplicada. 1981. Mapa Geológico do Estado de São) Paulo, escala 1: 500.000. Vol. I. São Paulo.

Marques-Toigo M.. Corrêa Z.C.S., Herter G.G. 1975. Geology and palinology of Candiota coal mine, RS, Brazil. In: Congreso Argentino de Paleontologia y Bioestratigrafia, 1, Tucumán, Actas, p. 401-427.

Millan, J.H. 1972. Macroflorula carbonífera de Monte Mor, Estado de São Paulo. Tese de Doutorado, Instituto de Geociências, Universidade de São Paulo, 154 pp.

Millan J.H. 1987. Os pisos florísticos do carvão do Subgrupo Itararé do Estado de São Paulo e suas implicações. In: SBP, Congr. Bras.
Paleont., 10. Rio de Janeiro, Anciis, 2:831-856.

Mune, S. \& Bernardes-de-Oliveira, M.E. 2002. Revisão das licófitas neocarboníferas do Sítio Volpe ex-Sítio da Mina), Município de Monte Mor, SP. - Porção inferior do Subgrupo Itararé, Grupo Tubarão, Brasil. Boletim Informativo da Sociedade Brasileira de Paleontologia, 40:20.

Pant D.D. \& Srivastava G.K. 1962. Structural studies of Lower Gondwana megaspores Part II. Specimens from Brazil and Mhukuru coalfielf, Tangayika. Palaeontographica Abt. B, 111:96-111.

Piérart P. 1959. Contribution à l'etude des espores et polens de la flore à Glossopteris contenus dans les charbons de La Luena (Katanga). Mém. Acad. Roy. Sc. Cl. Sc. Nat., 8:1-80.

Piérart P. 1975. Systématique, distribution stratigraphique et géographique des mégaspores du Carbonifére et du Permien. In: Congrés International de Stratigraphie et de Geológie du Carbon, 7. Kiefeld, Compte Rendu, 4:93-102.

Piérart P. 1981. Stratigraphical and géographical distribution of Gondwana megaspores. In: International Gondwana Symposium, 5. Wellington, Selected papers and abstracts, p. 19-21.

Piérart P. \& Dijkstra S. J. 1961. Étude comparée des mégaspores permiennes du Brésil et du Katanga. In: Congrés de stratigraphie et de Géologie du Carbonière, 4. Heerlen, Compte Rendu, 2:541-544.

Potonié R. 1956. Synopsis der Gattungen der Sporae dispersae I Teil: Sporites. Beih. Z. Geol. Jb. 23, 1035, IITaf, Hannover, 352 pp.

Ricardi-Branco F. 1998. Tafoflora Gondwanica do Membro Triunfo Formação Rio Bonito (Eopeniano), no Município de Figueira, PR. Tese de Doutoramento. Instituto de Geociências, Universidade de São Paulo, 182 pp.

Ricardi-Branco F., Arai M., Rösler O. 2002. Megaspores from coals of the Triunfo Member, Rio Bonito Formation (Lower Permian), northeastern Paraná State, Brazil. Ancis da Academia Brasileira de Ciências, 74:491-503.

Rocha-Campos A.C. \& Rösler O. 1978. Late Paleozoic faunal and floral sucessions in the Paraná basin, southeastern Brazil. Bol. IG-USP, 9: $1-15$.

Souza Filho E.E. de 1986. Mapeamento faciológico do Subgrupo Itararé na cuadrícula de Campinas (SP). Dissertação de Mestrado. Instituto de Geociências., Universidade de São Paulo, 121 pp.

Souza P.A. 2000. Palinobioestratigrafia do Subgrupo Itararé, Carbonífero/Permiano, na porção nordeste da Bacia do Paraná (SP/PR, Brasil). Tese de Doutoramento. Instituto de Geociências, Universidade de São Paulo, 299pp.

Spinner E. 1969. Preliminary study of the megaspores from the Tupe Formation, Quebrada del Tupe, La Rioja, Argentine. Pollen et Spores, 11: $669-685$.

Trindade N.M. 1959a. Megásporos gondwânicos de Charqueadas, Rio Grande do Sul. Boletim do DNPM, 194:1-35.

Trindade N.M. 1959b. O gênero Lagenoisporites no Gondwana brasileiro. Notas Preliminares e Estudos DNPM, 112:1-13. 
Trindade N.M. 1960. O gênero Triletes no Gondwana brasileiro. Notas Preliminares e Estudos DNPM, 114:1-17.

Trindade N.M. 1962. Megásporos gondwânicos da Mina do Leão, Rio Grande do Sul. Notas Preliminares e Estudos DNPM, 118:1-22.

Trindade N.M. 1964. Megásporos gondwânicos de Charqueadas, Rio Grande do Sul II. Boletim do DNPM, 26:1-27.

Trindade N.M. 1966. Megásporos gondwânicos da Mina de Arroio dos Ratos, Rio Grande do Sul. Notas Preliminares e Estudos DNPM, 133:1-28.

Trindade N.M. 1970. Megásporos carboníferos de Monte Mor, Estado de São Paulo. Anais Acad. Bras. Ciências, 42:459-470.

Trindade N.M. \& Sommer F.W. 1966. Sinopse sistemática dos megásporos do Gondwana Brasileiro. Anciis Acad. Bras. Ciências, 38:305-314.

Tyson R. 1995. Short course on palynofacies and organic facies. Ed. University of Newcastle. 50pp.

Manuscrito A-1425

Recebido em 15 de abril de 2003

Revisão dos autores em 10 de março de 2004 Revisão aceita em 20 de março de 2004 